\title{
Impulsive Systems triggered by Superposed Renewal Processes
}

\author{
Duarte Antunes, João P. Hespanha, and Carlos Silvestre
}

\begin{abstract}
We consider impulsive systems with several reset maps triggered by independent renewal processes, i.e., the intervals between jumps associated with a given reset map are identically distributed and independent of the other jump intervals. Considering linear dynamic and reset maps, we establish that mean exponential stability is equivalent to the spectral radius of an integral operator being less than one. The result builds upon a stochastic Lyapunov function approach which allows for providing stability conditions in the general case where the dynamic and the reset maps are non-linear. We also prove that the origin of an impulsive system with nonlinear dynamic and reset maps is stable with probability one if the linearization about zero equilibrium is mean exponentially stable, which justifies the importance of studying the linear case. The application of these results is illustrated in the context of networked control systems. The results in this paper permit the analysis of scenarios in which sensors transmit through independent communication links, which introduce independent and identically distributed intervals between transmissions, instead of sharing a single communication link, as considered in a previous work.
\end{abstract}

\section{INTRODUCTION}

We consider the following class of systems

$$
\begin{aligned}
& \dot{x}(t)=a(x(t)), \quad t \neq t_{k}^{l}, \quad t \geq 0, \\
& x\left(t_{k}^{l}\right)=j_{l}\left(x\left(t_{k}^{l-}\right)\right), \quad l \in \mathcal{L}, \quad x(0)=x_{0},
\end{aligned}
$$

where the state $x$ belongs to $\mathbb{R}^{n}$, the notation $x\left(t_{k}^{l-}\right)$ indicates the limit from the left of $x$ at the point $t_{k}^{l}$, and $\mathcal{L}:=$ $\left\{1, \ldots, n_{l}\right\}$. The reset maps $j_{l}$ are triggered at jump times $\left\{t_{k}^{l}, k>0, t_{0}^{l}:=0, l \in \mathcal{L}\right\}$, and the duration of the intervals $\left\{h_{k}^{l}=t_{k+1}^{l}-t_{k}^{l}, k \geq 0\right\}$ between jumps times associated with a given reset map $l$, are independent and identically distributed following a given distribution $F_{l}$ and also independent of the jump intervals $\left\{h_{k}^{j}, j \neq l, j \in \mathcal{L}\right\}$, associated with the other reset maps. The process that counts the number of jumps associated with a given reset map up to a given time $t$ is a renewal process, and the process that counts the total number of jumps is a superposed renewal process [1], [2]. This motivated us to call (1) an impulsive system triggered by superposed renewal processes.

The work of C.Silvestre and D.Antunes was partially supported by FCT (ISR/IST pluriannual funding) through the POS Conhecimento Program that includes FEDER funds, the PTDC/MAR/64546/2006 OBSERVFLY project, and the FCT PhD Student Scholarship, SFRH/BD/24632/2005.

The work of J. Hespanha was partially supported by the NSF Grants CNS-0720842, ECCS-0725485, and ECCS-0835847.

D. Antunes and C. Silvestre are with the Dep. of Electrical Eng. and Computer Science, Instituto Superior Técnico, ISR, 1046-001 Lisboa, Portugal. \{dantunes, cjs\}eisr.ist.utl.pt

João P. Hespanha is with the Dept. of Electrical and Compute Eng., University of California, Santa Barbara, CA 93106-9560, USA. hespanhadece.ucsb.edu
Our main motivation to consider such systems are networked control systems. Consider the case in which a remote controller communicates with a plant through two independent communication links; e.g., the actuation data is sent from the controller to the plant through a shared network and the sensor data is sent from the plant to the controller through a different shared network. A reasonable assumption in control over networks utilizing CSMA-type protocols [3] is to consider that the lengths of times that a user takes to gain access to the shared network and transmit data are independent and identically distributed. This networked control systems framework can be modeled by the system (1), where the map a models the plant and controller dynamics and the reset maps $j_{l}, l \in \mathcal{L}$ model the updates of received actuation and sensor data at transmission times, which are triggered by independent renewal processes. Several other scenarios in network control systems can be modeled using (1) and, in the present paper, we consider the case where two sensors transmit data to a controller through independent links that introduce independent and identically distributed intervals between transmissions.

Our main result establishes that when the maps $a$ and $j_{l}, l \in \mathcal{L}$ of the system (1) are linear, mean exponential stability is equivalent to the spectral radius of an integral operator being less than one. To prove this result, we show that the system (1) can be cast into the framework of piecewise deterministic processes [4], and derive conditions for mean exponential stability for (1) with general non-linear maps in terms of the extended generator given in [4]. When specialized to linear maps $a$ and $j_{l}, l \in \mathcal{L}$, this stability condition can be expressed in terms of the existence of a solution to an integro-differential equation, which, in turn, is related to the spectral radius of an integral operator.

An important motivation that lead us to study the linear version of (3) is provided by another contribution of this paper. Namely, we show that the origin of the system (1) is stable with probability one in the sense of [5] if the linearization of (1) about zero equilibrium is mean exponentially stable.

The applicability of the results of the present paper is illustrated through the stability analysis of networked control systems. We consider a benchmark example that appeared in [3], where we assumed that the sensors transmit in a round-robin fashion through a single shared link. We can now test mean exponential stability in the case where the sensors transmit through two independent, and therefore asynchronous, links both introducing independent and identically distributed intervals between transmissions.

Several references to related work on networked control 
systems can be found in [6]. Superposed renewal process are studied in [1] where one can find various applications including multi-server queues (see also [2]). Our results build upon the definition of piecewise deterministic processes [4], and also on the stochastic Lyapunov function approach provided in [5].

The remainder of the paper is organized as follows. In Section II we state our main results. In Section III we establish the connection between (1) and piecewise deterministic processes, which leads to the proof of our main result. An application example to networked control systems is discussed in Section IV. Conclusions are provided in Section V. Due to space limitations, some proofs are omitted, but can be found in [7].

Notation and Preliminaries: The functions $a$ and $j_{l}, l \in \mathcal{L}$ of the system (1) are assumed to be continuously differentiable and the origin is an equilibrium point, i.e., $a(0)=0$ and $j_{l}(0)=0, \forall_{l \in \mathcal{L}}$. We assume that $F_{l}(x)=\int_{0}^{x} f_{l}(t) d t$, $l \in \mathcal{L}$ where $f_{l}(t) \geq 0$ is a continuous probability density function, with finite support in the interval $\left[0, T_{l}\right], T_{l}>0$. We denote by $S_{l}(x)$ the survivor function of $F_{l}(x)$, i.e., $S_{l}(x)=1-F_{l}(x)$ and by $\lambda_{l}(x):=\frac{f_{l}(x)}{S_{l}(x)}$ the hazard rate. For a matrix $A, A^{\top}$ denotes the transpose, $A^{*}$ denotes the hermitian, and an eigenvalue is denoted by $\lambda_{j}(A)$. The identity matrix is denoted by $I$. We use $L_{2}\left(E, \mathbb{R}^{n}\right)$ to denote the space of equivalent classes of square Lebesgue integrable functions with domain on $E$ taking value on $\mathbb{R}^{n}$, endowed with the inner product $\langle f, g\rangle=\int_{E} g(x)^{*} f(x) d x$, for $f, g \in$ $L_{2}\left(E, \mathbb{R}^{n}\right)$, i.e., $\langle f, f\rangle<\infty$ and $\langle g, g\rangle<\infty$. The underlying probability space of the process defined by (1) is denoted by $(\boldsymbol{\Omega}, \boldsymbol{B}, \boldsymbol{P})$.

\section{MAin Results}

We say that the system (1) is mean exponentially stable (MES) if there exists constants $c>0$ and $\alpha>0$ such that for any initial condition $x_{0}$ of the system (1), we have that

$$
\mathbb{E}\left[x(t)^{\top} x(t)\right] \leq c e^{-\alpha t} x_{0}^{\top} x_{0}, \forall_{t \geq 0} .
$$

Consider the following linear version of (1)

$$
\begin{aligned}
& \dot{x}(t)=A x(t), \quad t \neq t_{k}^{l}, \quad t \geq 0 \\
& x\left(t_{k}^{l}\right)=J_{l} x\left(t_{k}^{l-}\right), \quad l \in \mathcal{L}, \quad x(0)=x_{0},
\end{aligned}
$$

where as in (1), $t_{0}^{l}:=0, l \in \mathcal{L}$, and the duration of the intervals $\left\{h_{k}^{l}=t_{k+1}^{l}-t_{k}^{l}, k \geq 0\right\}$ between jump times associated with a given reset map $l$, are independent and identically distributed following a given distribution $F_{l}$ and also independent of the jump intervals $\left\{h_{k}^{j}, j \neq l, j \in \mathcal{L}\right\}$, associated with the other reset maps.

For clarity of exposition, we start by considering the case of just two reset maps triggered by independent renewal processes, i.e., $n_{l}=2, \mathcal{L}=\{1,2\}$, and then present the general case in Subsection II-A. Consider the following matrix-valued $n \times n$ functions $P_{1}\left(\tau_{2}\right), \tau_{2} \in\left[0, T_{2}\right]$, and $P_{2}\left(\tau_{1}\right), \tau_{1} \in\left[0, T_{1}\right]$, where $T_{1}$ and $T_{2}$ are the support of the distributions $F_{1}$ and $F_{2}$ for the intervals between jumps, respectively, defined on $P_{1} \in L_{2}\left(\left[0, T_{2}\right], \mathbb{R}^{n \times n}\right)$ and $P_{2} \in$
$L_{2}\left(\left[0, T_{1}\right], \mathbb{R}^{n \times n}\right)$. Denoting by $\mathcal{P}$ the Cartesian product of two such functions $\left(P_{1}\left(\tau_{2}\right), P_{2}\left(\tau_{1}\right)\right) \in \mathcal{P}$, let $\mathfrak{L}: \mathcal{P} \mapsto \mathcal{P}$ be the following integral operator

$$
\left(Q_{1}, Q_{2}\right)=\mathfrak{L}\left(P_{1}, P_{2}\right),
$$

where

$$
\begin{aligned}
& Q_{1}\left(\tau_{2}\right)=\int_{0}^{\bar{T}_{1}}\left(J_{1} e^{A s}\right)^{\top} P_{1}\left(\tau_{2}+s\right) J_{1} e^{A s} \frac{S_{2}\left(\tau_{2}+s\right)}{S_{2}\left(\tau_{2}\right)} f_{1}(s) d s \\
&+\int_{0}^{\bar{T}_{1}}\left(J_{2} e^{A s}\right)^{\top} P_{2}(s) J_{2} e^{A s} S_{1}(s) \frac{f_{2}\left(\tau_{2}+s\right)}{S_{2}\left(\tau_{2}\right)} d s, \\
& \tau_{2} \in\left[0, T_{2}\right], \\
& Q_{2}\left(\tau_{1}\right)= \int_{0}^{\bar{T}_{2}}\left(J_{2} e^{A s}\right)^{\top} P_{2}\left(\tau_{1}+s\right) J_{2} e^{A s} \frac{S_{1}\left(\tau_{1}+s\right)}{S_{1}\left(\tau_{1}\right)} f_{2}(s) d s \\
&+\int_{0}^{\bar{T}_{2}}\left(J_{1} e^{A s}\right)^{\top} P_{1}(s) J_{1} e^{A s} S_{2}(s) \frac{f_{1}\left(\tau_{1}+s\right)}{S_{1}\left(\tau_{1}\right)} d s, \\
& \tau_{1} \in\left[0, T_{1}\right],
\end{aligned}
$$

$\bar{T}_{1}:=\min \left(T_{1}, T_{2}-\tau_{2}\right)$ and $\bar{T}_{2}:=\min \left(T_{1}-\tau_{1}, T_{2}\right)$; and $S_{1}(x):=1-F_{1}(x), S_{2}(x):=1-F_{2}(x)$ are the survivor functions of the distributions of the time intervals between resets. The following result provides a necessary and sufficient stability test for (3) with just two reset maps. Let $\sigma(\mathfrak{L})$ denote the spectral radius of the operator $\mathfrak{L}$.

Theorem 1: The system (3) with $n_{l}=2$ is MES if and only if $\sigma(\mathfrak{L})<1$, where $\mathfrak{L}$ is defined by (4).

Numerical efficient methods to compute the spectral radius of $\mathfrak{L}$ can be found in [8].

\section{A. Arbitrary number of reset maps}

The Theorem 1 can be extended to the case of an arbitrary number of jumps, by extending the definitions of the integral operator $\mathfrak{L}$. Let $B_{l}:=\left[0, T_{l}\right], \quad l \in \mathcal{L}$, where $T_{l}$ are the supports of the distributions between jump times, and

$$
B:=B_{1} \times \cdots \times B_{n_{l}} .
$$

Define a variable $\tau=\left(\tau_{1}, \tau_{2}, \ldots, \tau_{n_{l}}\right) \in B$ where $\tau_{j} \in$ $B_{j}$. Furthermore, let $\hat{B}_{j}=B_{1} \times \ldots B_{j-1} \times B_{j+1} \times \cdots \times$ $B_{n_{l}}$ and define the two following maps: $\pi_{l}$ that removes the component $l$ from the vector $\tau$, i.e., $\pi_{l}: B \mapsto \hat{B}_{l}, \pi_{l}(\tau)=$ $\left(\tau_{1}, \ldots, \tau_{l-1}, \tau_{l+1}, \ldots, \tau_{n_{l}}\right)$; and $\pi_{l}^{0}$ that sets the component $l$ of the vector $\pi$ to zero, i.e.,

$$
\pi_{l}^{0}: B \mapsto B, \pi_{l}^{0}(\tau)=\left(\tau_{1}, \ldots, \tau_{l-1}, 0, \tau_{l+1}, \ldots, \tau_{n_{l}}\right) .
$$

Let $\mathcal{P}$ be the space of $n_{l}$ matrix-valued $n \times n$ functions each belonging to $L_{2}\left(\hat{B}_{l}, \mathbb{R}^{n \times n}\right), l \in \mathcal{L}$, i.e., if $P \in \mathcal{P}$ then $P$ takes the form $P=\left(P_{1}\left(\hat{\tau}^{1}\right), \ldots, P_{n_{l}}\left(\hat{\tau}^{n_{l}}\right)\right)$ where $\hat{\tau}^{l} \in \hat{B}_{l}$ and $P_{l} \in L_{2}\left(\hat{B}_{l}, \mathbb{R}^{n \times n}\right)$. Then $\mathfrak{L}: \mathcal{P} \mapsto \mathcal{P}$ is an integral operator from $\mathcal{P}$ to itself defined as follows

$$
\left(Q_{1}, \ldots, Q_{n_{l}}\right)=\mathfrak{L}\left(P_{1}, \ldots, P_{n_{l}}\right)
$$

where $Q_{l}\left(\pi_{l}(\tau)\right):=R\left(\pi_{l}^{0}(\tau)\right)$,

$$
\begin{aligned}
& R(\tau):= \\
& \sum_{l=1}^{n_{l}} \int_{0}^{\bar{T}}\left(J_{l} e^{A s}\right)^{\top} P_{l}\left(\pi_{l}(\tau)+s 1_{n_{l}-1}\right) J_{l} e^{A s} \frac{\hat{S}_{l}(\tau, s)}{\bar{S}_{l}(\tau)} \frac{f_{l}\left(\tau_{l}+s\right)}{S_{l}\left(\tau_{l}\right)} d s,
\end{aligned}
$$


$\hat{S}_{l}(\tau, s):=\prod_{j=1, j \neq l}^{n_{l}} S_{j}\left(\tau_{j}+s\right), \bar{S}_{l}(\tau):=\prod_{j=1, j \neq l}^{n_{l}} S_{j}\left(\tau_{j}\right)$, $\bar{T}:=\min \left\{T_{l}-\tau_{l}, l \in \mathcal{L}\right\}$ and $1_{n_{l}-1}$ is a vector with $n_{l}-1$ components set to one.

Theorem 2: The system (3) is MES if and only if $\sigma(\mathfrak{L})<$ 1 where $\mathfrak{L}$ is defined by (7).

The theorem is proved in the Section III-B. Note that the Theorem 1 is a special case of the Theorem 2 with $n_{l}=2$.

\section{B. Local stability about zero equilibrium}

In this subsection we establish that the origin of (1) is stable with probability one if the linearization about zero equilibrium is mean exponentially stable, which justifies the importance of studying the linear case (Theorems 1 and 2). We shall need to consider the process

$$
\mathbf{x}(t)=(x(t), \tau(t))
$$

where $\tau$ keeps track of the time elapsed since the last jump associated with each of the reset maps, and is defined as

$$
\tau=\left(\tau_{1}, \ldots, \tau_{n}\right), \tau_{l}:=t-t_{k}^{l}, l \in \mathcal{L} .
$$

We show in the next section that the process (9) is a Piecewise Deterministic Process and hence a Markov process (cf. [4]). Note that, although we assumed for simplicity that for each $l \in \mathcal{L}$ the duration of the first intervals $h_{0}^{l}=t_{1}^{l}-t_{0}^{l}$ between jump times are distributed according to a density function $f_{l}(s), s \in\left[0, T_{l}\right]$, we can consider the more general case where the system (1) starts with a given initial condition $\tau_{0}=\left(\tau_{01}, \ldots, \tau_{0 n_{l}}\right)$. This means that we can consider that $h_{0}^{l}$ is distributed according to $\frac{f_{l}\left(\tau_{0 l}+s\right)}{S_{l}\left(\tau_{0 l}\right)}, s \in\left[0, T_{l}-\tau_{0 l}\right], l \in \mathcal{L}$.

Following [5], we say that the origin of the system (1) is stable with probability one if for any $\rho>0$ and $\epsilon>0$ there is a $\delta(\rho, \epsilon)>0$ such that, if $\left\|x_{0}\right\|<\delta(\rho, \epsilon)$ then for every $\tau_{0} \in B$,

$$
\mathbf{P}_{\left(x_{0}, \tau_{0}\right)}\left\{\sup _{\infty>t \geq 0}\|x(t)\| \geq \epsilon\right\} \leq \rho
$$

where $\mathbf{P}_{\left(x_{0}, \tau_{0}\right)}$ denotes the probability function of the Markov process defined by the system (1) started at $\left(x_{0}, \tau_{0}\right)$.

Theorem 3: If (3) is MES with $A=\frac{\partial}{\partial x} a(x)_{\left.\right|_{x=0}}$ and $J_{l}=$ $\frac{\partial}{\partial x} j_{l}(x)_{\mid x=0}, l \in \mathcal{L}$ where $a$ and $j$ are the non-linear maps in (1), then the origin of (1) is stable with probability one.

\section{Piecewise Deterministic Systems AND PRoof OF THE THEOREM 2}

We start by showing that the process (9) is a piecewise deterministic process (PDP) in the sense of [4]. A PDP is a process where randomness comes into play at fixed or random jump times $t_{k}^{p d}$ but there is no source of uncertainty between these times. Let $x_{p d}(t) \in X_{p d}$ denote the state of the PDP, where $X_{p d} \in \mathbb{R}^{n_{p d}}$ is the state-space. In a simplified form that suffices for the present paper, a PDP can be described by three ingredients: a deterministic flow $f_{p d}\left(x_{p d}\right)$ which describes the evolution of the state between jump times, i.e., $\dot{x}_{p d}(t)=f_{p d}\left(x_{p d}(t)\right), t \in\left[t_{k}^{p d}, t_{k+1}^{p d}\right)$; a transition jump rate $\lambda_{p d}\left(x_{p d}(t)\right)$ which determines the occurrences of the jump times; and a transition probability measure over the state-space $Q_{p d}\left(x_{p d}, d y\right)$ that characterizes the probability of a jump from a state to another state at jump times. As shown in [4], the PDP can be realized in the probability space $\left\{u_{k}^{1}, u_{k}^{2}, k \geq 0\right\}$ where $\left\{u_{k}^{1}\right\}$ and $\left\{u_{k}^{2}\right\}$ are mutually independent and identically distributed uniform random variables with support in the interval $[0,1]$. The execution of the PDP can be described by

Execution 4:

i) Set $k=0$ and $t_{0}^{p d}=0, x\left(t_{0}^{p d}\right)=x_{0}$.

ii) Obtain a realization of the uniform random variable $u_{k}^{1}$ and obtain $\delta_{k}$ from

$$
\delta_{k}=\inf \left\{t: e^{-\int_{0}^{t} \lambda_{p d}\left(\phi\left(x_{p d}\left(t_{k}^{p d}\right), s\right)\right) d s} \leq u_{k}^{1}\right\}
$$

where $\phi\left(x_{p d}\left(t_{k}^{p d}\right), s\right)$ is the flow at time $s$ of the system described by $\dot{x}_{p d}(t)=f\left(x_{p d}(t)\right)$ with initial condition $x_{p d}\left(t_{k}\right)$. Set $t_{k+1}^{p d}=t_{k}^{p d}+\delta_{k}$ and $x_{p d}(t)=$ $\phi\left(x_{p d}\left(t_{k}^{p d}\right), t-t_{k}^{p d}\right)$ in the interval $t \in\left[t_{k}^{p d}, t_{k+1}^{p d}\right)$.

(iii) Obtain a realization of a uniform random variable $u_{k}^{2}$ that determines the next state $x\left(t_{k+1}^{p d}\right)$ according to the probability density function $Q_{p d}\left(x\left(t_{k+1}^{p d^{-}}\right), d y\right)$.

(iv) Set $k=k+1$ and repeat the construction starting at step (ii).

In [4], an expression is given for the extended generator $\mathfrak{A}$ of a PDP and a characterization is given for its domain, which consists of the class of functions for which

$\mathbb{E}_{\mathbf{x}_{\mathbf{0}}}\left(V\left(x_{p d}(t)\right)\right)=V\left(x_{p d}(0)\right)+\mathbb{E}_{\mathbf{x}_{\mathbf{0}}} \int_{0}^{t} \mathfrak{A} V\left(x_{p d}(s)\right) d s, \forall_{t \geq 0}$,

where $\mathbb{E}_{\mathbf{x}_{0}}$ denotes expectation given that the process started at the initial state $\mathbf{x}_{0}=x_{p d}(0)$. We see from (13) that the extended generator for PDPs plays the role of a Lyapunov derivative for deterministic systems. The expression for the extended generator $\mathfrak{A}$ takes the form

$$
\begin{aligned}
\mathfrak{A} V\left(x_{p d}\right): & =\mathfrak{X} V\left(x_{p d}\right)+ \\
& \lambda_{p d}\left(x_{p d}\right)\left[\int_{X} V(y) Q_{p d}\left(x_{p d}, d y\right)-V\left(x_{p d}\right)\right],
\end{aligned}
$$

where $\mathfrak{X} V\left(x_{p d}\right):=\sum_{i=1}^{n_{p d}} \frac{\partial V\left(x_{p d}\right)}{\partial x_{p d i}} f_{i}\left(x_{p d}\right)$ (cf. [4], pp. 70). To cast the system (1) as a PDP, we set

(i) $x_{p d}=\mathbf{x}=(x, \tau)$ with dynamics

$$
\dot{x}_{p d}=f_{p d}\left(x_{p d}\right)=\left[\begin{array}{c}
\dot{x} \\
\dot{\tau}
\end{array}\right]=\left[\begin{array}{c}
f(x) \\
1
\end{array}\right] ;
$$

(ii) $\lambda_{p d}\left(x_{p d}(t)\right)=\sum_{l=1}^{n_{l}} \lambda_{l}\left(\tau_{l}\right)$

where $\lambda_{l}\left(\tau_{l}\right):=\frac{f_{l}\left(\tau_{l}\right)}{S_{l}\left(\tau_{l}\right)}, l \in \mathcal{L}$;

(iii) $Q_{p d}$ to be a discrete distribution such that

$$
\begin{aligned}
\mathbf{P}\left[\left(x\left(t_{k}\right), \tau\left(t_{k}\right)\right)\right. & \left.=\left(J_{l} x\left(t_{k}^{-}\right), \pi_{0}^{l}\left(\tau\left(t_{k}^{-}\right)\right) \mid \mathbf{x}\left(t_{k}^{-}\right) \wedge E\right)\right] \\
& =\frac{\lambda_{l}\left(\tau_{l}\right)}{\sum_{l=1}^{n_{l}} \lambda_{l}\left(\tau_{l}\right)},
\end{aligned}
$$

where $E$ is the event of a jump occurring at time $t_{k}^{-}$. 
As stated next, the process defined by (1) can be simulated by the Execution 4 of this PDP.

Proposition 5: The stochastic process defined by the system (1) can be realized in the probability space of the PDP defined by the Execution 4 with $f_{p d}, \lambda_{p d}$, and $Q_{p d}$ defined by (15), (16), and (17).

The proof is omitted since it follows from the same arguments found in [4, pp.86,87]. Replacing (15), (16), and (17) in (18) we obtain the extended generator for (1):

$$
\begin{aligned}
\mathfrak{A} V(\mathbf{x}):= & \frac{\partial}{\partial \tau} V(\mathbf{x})+ \\
& \mathfrak{X}_{x} V(\mathbf{x})+\sum_{l=1}^{n_{l}} \lambda_{l}\left(\tau_{l}\right)\left[V\left(\left(j_{l}(x), \pi_{l}^{0}(\tau)\right)\right)-V(\mathbf{x})\right],
\end{aligned}
$$

where $\mathfrak{X}_{x} V(\mathbf{x}):=\sum_{i=1}^{n} \frac{\partial V(\mathbf{x})}{\partial x_{i}} f_{i}(x)$ and $\pi_{l}^{0}(\tau)$ is defined by (6).

\section{A. Mean exponential stability}

The following result, is a stochastic analog of a well known result for deterministic non-linear systems (cf. [9]).

Theorem 6: The system (1) is MES if and only if there exists a differentiable positive function $V(\mathbf{x})$ which equals zero at zero, and positive constants $c_{1}, c_{2}, k$ such that for every $\mathbf{x}=(x, \tau) \in \mathbb{R}^{n} \times B$,

$$
\begin{array}{r}
c_{1}\|x\|^{2} \leq V(\mathbf{x}) \leq c_{2}\|x\|^{2} \\
\mathfrak{A} V(\mathbf{x}) \leq-k\|x\|^{2}
\end{array}
$$

where $\mathfrak{A} V(\mathbf{x})$ is given by (18) and the set $B$ is described by $(5)$.

The Theorem 6 can be specialized to (3) as follows.

Theorem 7: The system (3) is MES if and only if for every $a_{1} I<Y(\tau)<a_{2} I, \forall \tau$ and $b_{1} I<Z(\tau)<b_{2} I, \forall \tau$ there exists $c_{1} I<X(\tau)<c_{2} I, \forall \tau$ such that for every $\tau \in B$,

$$
\begin{aligned}
& \sum_{l=1}^{n_{l}} \frac{\partial}{\partial \tau_{l}} X(\tau)+A^{\top} X(\tau)+X(\tau) A+ \\
& \quad \sum_{l=1}^{n_{l}} \lambda_{l}\left(\tau_{l}\right)\left[J_{l}^{\top} X\left(\pi_{l}^{0}(\tau)\right) J_{l}-X(\tau)+Z(\tau)\right]+Y(\tau)=0
\end{aligned}
$$

where $\pi_{l}^{0}$ is defined by (6), $a_{i}, b_{i}, c_{i}, i \in\{1,2\}$ are positive constants, and $X(\tau), Y(\tau), Z(\tau)$ are differentiable functions.

Remark 8: Although the proof of Theorem 7 is omitted we shall need the fact established in its proof that if the system (3) is MES if and only if there exists a bounded positive-definite matrix function $X(\tau)$ that is the solution to $(21)$, and is given by

$$
X(\tau)=X_{1}(\tau)+X_{2}(\tau)
$$

where

$$
\begin{aligned}
X_{1}(\tau) & :=\int_{0}^{+\infty} \mathbb{E}_{\tau}\left[\Phi(t)^{\top} Y(\tau) \Phi(t)\right] d t \\
X_{2}(\tau) & :=\sum_{k>0, l \in \mathcal{L}} \mathbb{E}_{\tau}\left[\Phi\left(t_{k}^{l}\right)^{\top} Z(\tau) \Phi\left(t_{k}^{l}\right)\right]
\end{aligned}
$$

$\Phi(t)$ is the transition matrix of the system (3), i.e.,

$$
\Phi(t)=e^{A\left(t-t_{r}^{l_{r}}\right)} J_{l_{r-1}} \ldots J_{l_{1}} e^{A h_{1}^{l_{1}}} J_{l_{0}} e^{A h_{0}^{l_{0}}},
$$

where $\left\{l_{j} \in \mathcal{L}, j \geq 0\right\}$ is the triggered sequence of reset maps, $r=\max \left\{k: t_{k} \leq t\right\}$ and $\mathbb{E}_{\tau}$ denotes expectation of the process $\Phi(t)$ started at an initial condition $\tau$.

\section{B. Proof of the Theorem (2)}

The proof will be established through a series of Lemmas.

Lemma 9: If $X(\tau)$, defined by (22), is bounded, then it satisfies

$X(\tau)=W(\tau)+$

$\sum_{l=1}^{n_{l}} \int_{0}^{T_{l}}\left(J_{l} e^{A s}\right)^{\top} X\left(\pi_{l}^{0}\left(\tau+s 1_{n_{l}}\right)\right) J_{l} e^{A s} \frac{\hat{S}_{l}(\tau, s)}{\bar{S}_{l}(\tau)} \frac{f_{l}\left(\tau_{l}+s\right)}{S_{l}\left(\tau_{l}\right)} d s$

where

$$
\begin{aligned}
W(\tau):= & \sum_{l=1}^{n_{l}} \int_{0}^{\bar{T}} e^{A^{\top} s} Z(\tau) e \frac{\hat{S}_{l}(\tau, s)}{\bar{S}_{l}(\tau)} \frac{f_{l}\left(\tau_{l}+s\right)}{S_{l}\left(\tau_{l}\right)} d s+ \\
& \int_{0}^{\bar{T}} e^{A^{\top} s} Y(\tau) e^{A s} \Pi_{l=1}^{n_{l}} \frac{S_{l}\left(\tau_{l}+s\right)}{S_{l}\left(\tau_{l}\right)} d s
\end{aligned}
$$

Proof: Denote by $t_{1}$ the time at which the first reset map is triggered, which can correspond to any of the $n_{l}$ reset maps. For a given time $t$ we can partition the probability space $\boldsymbol{\Omega}$ into $\boldsymbol{\Omega}=\left[t_{1} \leq t\right] \cup\left[t_{1}>t\right]$. We can further partition $\left[t_{1} \leq t\right]=\cup_{l=1}^{n_{l}} C_{l}(t)$ where $C_{l}(t)$ is the event of the transition $l$ being the first to trigger in the interval $[0, t]$, i.e., $C_{l}(t)=\left[\min \left\{\bar{h}^{j}, j \in \mathcal{L}\right\}=\bar{h}^{l}=t_{1} \leq\right.$ $t] \wedge\left[\bar{h}^{l}>\bar{h}^{j}, l \neq j\right]$, where $\bar{h}^{l}$ is distributed according to $\frac{f_{l}\left(\tau_{l}+s\right)}{S_{l}\left(\tau_{l}\right)}, s \in\left[0, T_{l}-\tau_{l}\right)$, for a given $l \in \mathcal{L}$, from the fact that the expected value is taken with respect to the process started at $\tau=\left(\tau_{1}, \tau_{2}, \ldots, \tau_{n l}\right)$. Using this partition we have

$$
\begin{aligned}
\mathbb{E}_{\tau}\left[\Phi(t)^{\top} Y(\tau) \Phi(t)\right] & =\mathbb{E}_{\tau}\left[\left(\Phi(t)^{\top} Y(\tau) \Phi(t)\right) \mathbf{1}_{\left[t_{1}>t\right]}\right] \\
& +\sum_{l=1}^{n_{l}} \mathbb{E}_{\tau}\left[\left(\Phi(t)^{\top} Y(\tau) \Phi(t)\right) \mathbf{1}_{C_{l}(t)}\right]
\end{aligned}
$$

The first term on the right hand side of (27) is given by

$\mathbb{E}_{\tau}\left[\Phi(t)^{\top} Y(\tau) \Phi(t) \mathbf{1}_{\left[t_{1}>t\right]}\right]=e^{A^{\top} t} Y(\tau) e^{A t} \Pi_{l=1}^{n_{l}} \frac{S_{l}\left(\tau_{l}+t\right)}{S_{l}\left(\tau_{l}\right)}$,

where we used the fact that $\mathbb{E}_{\tau}\left[\mathbf{1}_{\left[t_{1}>t\right]}\right]=\mathbf{P}\left(\left[t_{1}>t\right]\right)=$ $\prod_{l=1}^{n_{l}} \mathbf{P}\left[\bar{h}^{l}>t\right]=\Pi_{l=1}^{n_{l}} \frac{S_{l}\left(\tau_{l}+t\right)}{S_{l}\left(\tau_{l}\right)}$. To obtain an expression for the second term on the right hand side of (27), notice first that for a function of the first jump time $G\left(t_{1}\right)$,

$$
\begin{aligned}
\mathbb{E}_{\tau}\left[G\left(t_{1}\right) \mathbf{1}_{C_{l}(t)}\right] & =\int_{0}^{t} \mathbb{E}\left[G(s) \mathbf{1}_{\left[h^{j}>s, l \neq j\right]} \mid \bar{h}^{l}=s\right] \frac{f_{l}\left(\tau_{l}+s\right)}{S_{l}\left(\tau_{l}\right)} d s \\
& =\int_{0}^{t} G(s) \frac{\hat{S}_{l}(\tau, s)}{\bar{S}_{l}(\tau)} \frac{f_{l}\left(\tau_{l}+s\right)}{S_{l}\left(\tau_{l}\right)} d s
\end{aligned}
$$

Notice also that $\Phi(t)=\hat{\Phi}_{l}\left(t-t_{1}\right)\left(J_{l} e^{A t_{1}}\right)$ when the transition $l \in \mathcal{L}$ is first triggered, where $\hat{\Phi}_{l}\left(t-t_{1}\right)$ is the transition matrix from $t_{1}$ to $t$ starting the process at $\pi_{l}^{0}\left(\tau+s 1_{n_{l}}\right)$ where 
$\pi_{l}^{0}$ is defined by (6) and $1_{n_{l}}$ is a vector with $n_{l}$ components equal to one. Each of the terms of the summation on the right hand side of (27) can then be expressed as

$$
\begin{aligned}
& \mathbb{E}_{\tau}\left[\Phi(t)^{\top} Y(\tau) \Phi(t) \mathbf{1}_{C_{l}(t)}\right]= \\
& \int_{0}^{t}\left(J_{l} e^{A s}\right)^{\top} \mathbb{E}_{\pi_{l}^{0}\left(\tau+s 1_{n_{l}}\right)}\left[\hat{\Phi}(t-s)^{\top} Y(\tau) \hat{\Phi}(t-s)\right] J_{l} e^{A s} \alpha_{l}(\tau, s) d s
\end{aligned}
$$

where $\alpha_{l}(\tau, s):=\frac{\hat{S}_{l}(\tau, s)}{\bar{S}_{l}(\tau)} \frac{f_{l}\left(\tau_{l}+s\right)}{S_{l}\left(\tau_{l}\right)}$. By construction of the process

$$
\begin{aligned}
\mathbb{E}_{\pi_{l}^{0}\left(\tau+s 1_{n_{l}}\right)}\left[\left(\hat{\Phi}_{l}(t-s)^{\top} Y(\tau) \hat{\Phi}_{l}(t-s)\right]=\right. \\
\mathbb{E}_{\pi_{l}^{0}\left(\tau+s 1_{n_{l}}\right)}\left[\Phi(t-s)^{\top} Y(\tau) \Phi(t-s)\right] .
\end{aligned}
$$

Replacing (29) in (27) and (27) in (23) we obtain

$$
X_{1}(\tau)=\sum_{l=1}^{n_{l}} X_{1}^{l}(\tau)+\int_{0}^{\infty} e^{A^{\top} t} Y(\tau) e^{A t} \Pi_{l=1}^{n_{l}} \frac{S_{l}\left(\tau_{l}+t\right)}{S_{l}\left(\tau_{l}\right)} d t
$$

where

$$
\begin{aligned}
& X_{1}^{l}(\tau)= \\
& \int_{0}^{\infty} \int_{0}^{t} \mathbb{E}_{\pi_{l}^{0}\left(\tau+s 1_{n_{l}}\right)}\left[\left(J_{l} e^{A s}\right)^{\top}\left(\Phi(t-s)^{\top} Y(\tau) \Phi(t-s)\left(J_{l} e^{A s}\right) \alpha(\tau, s)\right] d s d t\right.
\end{aligned}
$$

Changing the order of integration in (32) we have that (31) can be written as

$$
\begin{aligned}
& X_{1}(\tau)=\int_{0}^{\bar{T}} e^{A^{\top} t} Y(\tau) e^{A t} \Pi_{l=1}^{n_{l}} \frac{S_{l}\left(\tau_{l}+t\right)}{S_{l}\left(\tau_{l}\right)} d t \\
& \sum_{l=1}^{n_{l}} \int_{0}^{\bar{T}}\left(J_{l} e^{A s}\right)^{\top} X_{1}\left(\pi_{l}^{0}\left(\tau+s 1_{n_{l}}\right)\right) J_{l} e^{A s} \frac{\hat{S}_{l}\left(\tau_{l}, s\right)}{\bar{S}_{l}\left(\tau_{l}\right)} \frac{f_{l}\left(\tau_{l}+s\right)}{S_{l}\left(\tau_{l}\right)} d s
\end{aligned}
$$

With similar computations one can conclude that for $t_{k}^{l}>$ $t_{1}, k \geq 2$ we have that

$$
\begin{aligned}
& \mathbb{E}_{\tau}\left[\Phi\left(t_{k}^{l}\right)^{\top} Z(\tau) \Phi\left(t_{k}^{l}\right) \mathbf{1}_{C_{l}(t)}\right]= \\
& \int_{0}^{\bar{T}}\left[( J _ { l } e ^ { A s } ) ^ { \top } \mathbb { E } _ { \pi _ { l } ^ { 0 } ( \tau + s 1 _ { n _ { l } } ) } \left[\left(\Phi\left(t_{k-1}^{l}\right)^{\top} Z(\tau) \Phi\left(t_{k-1}^{l}\right)\right] J_{l} e^{A s} \alpha_{l}(\tau, s) d s,\right.\right.
\end{aligned}
$$

and that

$$
\begin{aligned}
& X_{2}(\tau)=\sum_{l=1}^{n_{l}} \int_{0}^{\bar{T}} e^{A^{\top} s} Z(\tau) e^{A s} \frac{\hat{S}_{l}(\tau, s)}{\bar{S}_{l}(\tau)} \frac{f_{l}\left(\tau_{l}+s\right)}{S_{l}\left(\tau_{l}\right)} d s+ \\
& \sum_{l=1}^{n_{l}} \int_{0}^{\bar{T}}\left(J_{l} e^{A s}\right)^{\top} X_{2}\left(\tau_{l}^{0}\left(\tau+s 1_{n_{l}}\right)\right) J_{l} e^{A s} \frac{\hat{S}_{l}(\tau, s)}{\bar{S}_{l}(\tau)} \frac{f_{l}\left(\tau_{l}+s\right)}{S_{l}\left(\tau_{l}\right)} d s
\end{aligned}
$$

Since $X(\tau)=X_{1}(\tau)+X_{2}(\tau)$ adding (33) and (34) we obtain (26).

Let

$$
P\left(\pi_{l}(\tau)\right):=X\left(\pi_{l}^{0}(\tau)\right), \quad \pi_{l}(\tau) \in B_{l} .
$$

Lemma 10: The function $P$ defined by (35) satisfies the Fredholm equation

$$
P=\mathfrak{L}(P)+U
$$

where $\mathfrak{L}(P)$ is defined by (7), and where $U:=$ $\left(U_{1}\left(\pi_{1}(\tau)\right), \ldots, U_{n_{l}}\left(\pi_{n_{l}}(\tau)\right)\right), U_{l}\left(\pi_{l}(\tau)\right):=W\left(\pi_{l}^{0}(\tau)\right), l \in \mathcal{L}$.

Proof: Obtained by directly replacing (35) in (26).

The next result is key for what follows. Let $\mathcal{V}$ be the space of elements of $\left(V_{1}\left(\hat{\tau}_{1}\right), \ldots, V_{n_{l}}\left(\hat{\tau}_{n_{l}}\right)\right) \in \mathcal{P}$ for which $V_{l}\left(\hat{\tau}_{l}\right) \geq 0, \forall_{l \in \mathfrak{L}}, \forall_{\hat{\tau}_{l} \in B_{l}}$, and $\mathfrak{L}^{\prime}$ denote the adjoint operator of $\mathfrak{L}$. The inner product of two elements $U=\left(U_{1}, \ldots, U_{n_{l}}\right), V=\left(V_{1}, \ldots, V_{n_{l}}\right) \in \mathcal{V}$ is defined as $\langle U, V\rangle=\sum_{i=1}^{n_{l}}\left\langle U_{i}, V_{i}\right\rangle$.

Lemma 11: There exists $V \in \mathcal{V}$ such that $\mathfrak{L}^{\prime}(V)=$ $\sigma(L) V$

The proof follows from establishing that $\mathcal{V}$ is a cone, $\mathfrak{L}^{\prime}$ and $\mathfrak{L}^{\prime}$ are positive operators [10] with respect to $\mathcal{V}$, and using [10, Thm. 9.2] which states that a positive operator has an eigenvalue that equals the spectral radius and an eigenvector that belongs to the cone. The full derivation is however omitted due to space limitations.

Lemma 12: The equation (36) has a bounded solution $P \in$ $\mathcal{V}$ if and only if $\sigma(\mathfrak{L})<1$.

Proof: Sufficiency follows from the fact that if $\sigma(\mathfrak{L})<$ 1 then $P=\sum_{i=0}^{\infty} \mathfrak{L}^{i}(U)$ exists which is the solution to $P=\mathfrak{L}(P)+U$, where $\mathfrak{L}^{i}$ denotes the composite operator obtained by applying $i$ times $\mathfrak{L}$, e.g., $\mathfrak{L}^{2}(P)=\mathfrak{L}(\mathfrak{L}(P))$ and $\mathfrak{L}^{0}(P):=P$. Since $\mathfrak{L}$ is a positive operator with respect to $\mathcal{V}$, i.e., $L(V) \in \mathcal{V}$ if $V \in \mathcal{V}$ then $\mathfrak{L}^{i}(U) \in \mathcal{V}, \forall_{i \geq 0}$. Thus $P \in \mathcal{V}$ since $P$ is a sum of elements in $\mathcal{V}$.

To prove necessity, suppose that $\sigma(\mathfrak{L}) \geq 1$ and (36) has a solution $P \in \mathcal{V}$. Then $\sigma\left(\mathfrak{L}^{*}\right)=\sigma(\mathfrak{L}) \geq 1$. From Lemma 11 there exists $R \in \mathcal{V}$ such that

$$
\mathfrak{L}^{*}(R)=\sigma\left(\mathfrak{L}^{*}\right) R .
$$

Taking the inner product with $R$ on both sides of (36) yields

$$
\begin{aligned}
& \langle R, P\rangle=\langle R, \mathfrak{L}(P)\rangle+\langle R, U\rangle \Leftrightarrow\langle R, P\rangle=\left\langle\mathfrak{L}^{*}(R), P\right\rangle+\langle R, U\rangle \\
& \Leftrightarrow\langle R, P\rangle\left(1-\sigma\left(\mathfrak{L}^{*}\right)\right)=\langle R, U\rangle
\end{aligned}
$$

Now $\langle R, P\rangle \geq 0$, and $\langle R, U\rangle>0$, since $R$ is different from the zero element in $\mathcal{V}$ and $U=\left(U_{1}, \ldots, U_{n_{l}}\right) \in \mathcal{V}$ is such $U_{l}(\tau)>0, \forall_{l \in \mathcal{L}, \tau \in\left[0, T_{\min }\right)}$ and $T_{\min }=\min \left\{T_{l}\right\}$. Thus, from (37) we conclude that $\sigma\left(\mathfrak{L}^{*}\right)=\sigma(\mathfrak{L}) \geq 1$ leads to a contradiction.

We now prove the Theorem 2.

Proof: (of Thereom 2) Note that, MES of the system (1), is equivalent to (22) being bounded (cf. Remark 8). If (22) is bounded, from the Lemma 9 and (35) we have that $P \in \mathcal{V}$, and from the Lemma 9, we have that $P \in \mathcal{V}$ is the solution to (36). Conversely if (36) has a bounded solution $P \in \mathcal{V}$ then (26) is bounded and therefore (22) is bounded. From the Lemma 12 the existence of a solution $P \in \mathcal{V}$ to (36) is equivalent to $\sigma(\mathfrak{L})<1$.

\section{Application to Networked Control}

In [3] a networked control system is considered, in which a plant and a controller are connected through a communication network. The linear plant and linear controller take the form:

$$
\dot{x}_{P}=A_{P} x_{P}+B_{P} u, \quad\left[\begin{array}{l}
y_{1} \\
y_{2}
\end{array}\right]=\left[\begin{array}{l}
C_{P 1} \\
C_{P 2}
\end{array}\right] x_{P}
$$

$$
\dot{x}_{C}=A_{C} x_{C}+\left[\begin{array}{ll}
B_{C 1} & B_{C 2}
\end{array}\right]\left[\begin{array}{l}
\hat{y}_{1} \\
\hat{y}_{2}
\end{array}\right], u=C_{C} x_{C}+\left[\begin{array}{ll}
D_{C 1} & D_{C 2}
\end{array}\right]\left[\begin{array}{l}
\hat{y}_{1} \\
\hat{y}_{2}
\end{array}\right]
$$


where $x_{P} \in \mathbb{R}^{n_{p}}$ and $x_{C} \in \mathbb{R}^{n_{c}}$ are the states of the plant and of the controller. The continuous-time actuation $u(t)$ is directly applied to the plant, but the two output vectors of the plant $\left(y_{1}, y_{2}\right)$ are sent through the communication network, and are held constant at the controller side, i.e.,

$$
\begin{aligned}
& \hat{y}_{1}(t)=\hat{y}_{1}\left(t_{k}^{1}\right)=y_{1}\left(t_{k}^{1}\right), t \in\left[t_{k}^{1}, t_{k+1}^{1}\right), \\
& \hat{y}_{2}(t)=\hat{y}_{2}\left(t_{k}^{2}\right)=y_{2}\left(t_{k}^{2}\right), t \in\left[t_{k}^{2}, t_{k+1}^{2}\right), k \in \mathbb{Z}_{\geq 0},
\end{aligned}
$$

where $t_{k}^{l}$ are the times at which the output $y_{l}$ is sent, $l \in$ $\{1,2\}$. Let $e_{y}:=\hat{y}-y$. The dynamic equations for $x:=$ $\left[\begin{array}{lll}x_{P}^{\top} & x_{C}^{\top} & e_{y}^{\top}\end{array}\right]^{\top}$ take the form

$$
\begin{aligned}
& \dot{x}(t)=A x(t), \quad t \neq t_{k}^{l} \\
& x\left(t_{k}^{l}\right)=J_{l} x\left(t_{k}^{l-}\right), \quad l \in\{1,2\}
\end{aligned}
$$

where

$A=\left[\begin{array}{ll}A_{x x} & A_{x e} \\ A_{e x} & A_{e e}\end{array}\right], J_{1}=\left[\begin{array}{cc}I_{n_{p}+n_{c}} & 0 \\ 0 & R_{1}\end{array}\right], J_{2}=\left[\begin{array}{cc}I_{n_{p}+n_{c}} & 0 \\ 0 & R_{2}\end{array}\right]$

and

$$
\begin{aligned}
A_{x x} & =\left[\begin{array}{cc}
A_{P}+B_{P} D_{C} C_{P} & B_{P} C_{C} \\
B_{C} C_{P} & A_{C}
\end{array}\right], A_{x e}=\left[\begin{array}{c}
B_{P} D_{C} \\
B_{C}
\end{array}\right], \\
A_{e x} & =\left[\begin{array}{ll}
-C_{P} & 0
\end{array}\right] A_{x x}, \quad A_{e e}=\left[\begin{array}{ll}
-C_{P} & 0
\end{array}\right] A_{x e}, \\
R_{1} & =\left[\begin{array}{ll}
0 & 0 \\
0 & 1
\end{array}\right], \text { and } \quad R_{2}=\left[\begin{array}{ll}
1 & 0 \\
0 & 0
\end{array}\right] .
\end{aligned}
$$

In [3], it is assumed that the outputs are sent in a round-robin fashion through a single shared communication network. When the distribution of the intervals between consecutive transmissions is assumed to be, e.g., uniform with a support $T$, we can use the results in [3] to study the stability of this system.

Suppose now that, instead of transmitting the two measurements in a round robin fashion through the same communication network, the two sensors transmit data through two independent communication links. We assume that both links are shared with other users and that the intervals between consecutive transmissions can be modeled by independent processes with support in the interval $\left[0, T_{l}\right]$ for the link associated with the output $y_{l}, l \in\{1,2\}$. We compare next the results of using a single link with a round-robin protocol, and two independent links for two benchmark problems.

\section{A. Batch Reactor}

This example considers the control of a linearized model of an open loop unstable two-input two-output batch reactor, described by (38), where the expressions for $\left(A_{P}, B_{P}, C_{P}\right)$ can be found in [3]. The system is controlled by a PI controller, described by (39), where the expression for $\left(A_{C}, B_{C}, C_{C}, D_{C}\right)$ can be found in [3].

For the case of two independent links we take a grid of values of $T_{1}$ and $T_{2}$, i.e., the support of the distributions between transmissions, and test mean exponential stability of the closed-loop using a numerical method taken from [8] to compute the spectral radius of the operator defined in the Theorem 1. The results obtained are summarized in the Figure 1. If the distributions of the two links have the same

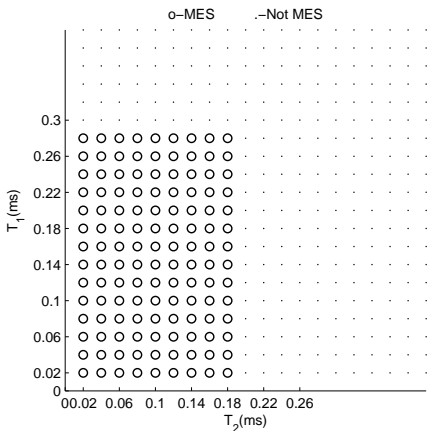

Fig. 1. MES for various values of the support of a uniform distributions of the transmission intervals of two independent links.

support then stability is preserved for every $T_{1} \in[0, \bar{T}]$ and $T_{2} \in[0, \bar{T}]$, where $\bar{T}=0.18$. For a round-robin singlelink protocol the maximum support obtained in [3] is $T=$ 0.11 . This means that consecutive transmissions of the same output are distributed according to a triangular distribution with support $T=0.22$, but note that in this case the duration of the intervals between transmissions of the two outputs are not independent, and a different approach must be used to assert stability (see [3]). Thus, when the two links have the same support, the fact that $T$ and $\bar{T}$ are close fits well with the results obtained previously in [3]. If the two links have different supports one can conclude from the Figure 1 that the mean exponential stability of the closed-loop is lost for a lower value of the support of the distributions associated with the output $y_{2}$ than the value of the support associated with the output $y_{1}$.

\section{CONCLUSIONS}

We studied impulsive systems triggered by superposed renewal processes, motivated by their applications to networked control. A topic for future work is to consider possible atom points and infinite support for the distributions for the intervals between resets.

\section{REFERENCES}

[1] C. Lam and J. Lehoczky, "Superposition of renewal processes," Adv. in Appl. Probab., no. 23, p. 6485, 1991.

[2] O. Kella and W. Stadje, "Superposition of renewal processes and an application to multi-server queues," Statistics \& Probability Letters, vol. 76, no. 17, pp. 1914 - 1924, 2006.

[3] D. Antunes, J. P. Hespanha, and C. Silvestre, "Stability of impulsive systems driven by renewal processes," in American Control Conference, 2009. ACC '09., June 2009, pp. 4032-4037.

[4] M. H. A. Davis, Markov Models and Optimization. London, UK: Chapman \& Hall, 1993.

[5] H. J. Kushner, Stochastic stability and control [by] Harold J. Kushner. Academic Press, New York,, 1967.

[6] J. P. Hespanha, P. Naghshtabrizi, and Y. Xu, "A survey of recent results in networked control systems," Proc. of IEEE Special Issue on Technology of Networked Control Systems, vol. 95, no. 1, pp. 138-162, Jan. 2007.

[7] D. Antunes, J. P. Hespanha, and C. Silvestre, "Impulsive systems triggered by superposed renewal processes," IST, Tech. Rep., 2010, available at http://users.isr.ist.utl.pt/ $\sim$ dantunes/.

[8] F. Chatelin, Spectral approximation of linear operators. Academic Press, 1983.

[9] H. Khalil, Nonlinear Systems, Third Edition. Upper Saddle River, NJ: Prentice Hall, 2000.

[10] M. A. Krasnoselskii, J. A. Lifshits, and A. V. Sobolev, Positive linear systems : the method of positive operators. Berlin: Heldermann, Verlag, 1989. 